\title{
Bounding the Probability of Failure for Levee Systems
}

\author{
Justin C. Hollenback and Robb E. S. Moss
}

\begin{abstract}
An exact solution for the probability of failure of large complex infrastructure systems is rarely obtainable; however the probability of failure can often be bounded. An example of this type of system is the levee system in the California Bay Delta. Large levee systems often consist of many components arranged in series and parallel sub-systems. There is the problem of defining component (or reach) length, and therefore the total number of components in the system where component length is dependent on failure mode. Methods of bounding probability of failure based on uni-, bi-, and tri-modal component probability of failure are discussed. The bounds are highly sensitive to the total number of components in the system. Characterization of spatial variability using semi-variograms is used to define component length for various failure modes. Combining the statistically defined component length with system probability of failure bounds allow for a more accurate estimate of failure probability. Demonstration of these methods and results for specific levee systems in the California Bay Delta are shown in this paper.
\end{abstract}

\section{INTRODUCTION}

Society is dependent on a wide variety of complex systems (e.g., water and power distribution). As time progresses the likelihood that a system will be exposed to hazards increases. In general, a system's ability to survive exposures decrease; a system tends to fatigue and degrade with time. Also with time a system can become increasingly complex. When a complex system is due for repair and improvements, constraints on logistics, resources, and funding make it nearly impossible for the entire system to be repaired at once. Thus, repair on specific components need to be prioritized so critical components get repaired first. This situation lends itself to the implementation of risk analysis; risk being the product of failure probability for a component and consequences of that failure, where failure probability is usually annualized and consequences are in terms of cost, lives lost, etc. The benefit of using risk to make critical decisions is in its ability to bring both the likelihood of component failure and the detrimental effects of component failure into one metric.

Our research focuses on levee systems, specifically the California Bay Delta and its use as a water distribution hub. The Bay Delta consists of a network of channels confined by a system of more than $1700 \mathrm{~km}$ of levees. These levees protect a collection of 65 islands and tracts, many of which have landside elevations below sea level. This means levees surrounding these islands hold back water year round, 
even during dry periods. Roughly $25 \%$ of urban water used in the state is diverted through the Bay Delta. Approximately two thirds of the state's population relies on the Bay Delta for some portion of their drinking water and nearly 3 million acres of farmland depend on the Bay Delta for some quantity of irrigation water. The Bay Delta is currently in a fragile state. A major earthquake in the bay area could result in the failure of hundreds of kilometers of levees. This would, among other things, result in saltwater contamination of fresh water in the delta, rendering it useless as a fresh water distribution hub for a period of months, possibly years (URS, 2008).

Calculating the probability of failure for large complex systems, like the Bay Delta levees, is non-trivial and exact solutions are often unattainable for practical purposes. Bounding the probability of failure is a reasonable alternative to exact solutions. Probability of failure, exact solution or bounded, is sensitive to the number of components present in the system. Currently, the number of components in a levee system is not robustly defined. Here we review procedures for bounding the probability of failure for systems and present a method for statistically defining the number of components in a levee system.

\section{BOUNDS ON PROBABILITY OF FAILURE FOR LARGE SYSTEMS}

In civil engineering complex systems are generally composed of two types of idealized two-state sub-systems, parallel and series. A two-state system is either in survival state or failure state. Parallel systems, or redundant systems, are in failure state if all components fail. Series systems, or non-redundant systems, are in failure state if one or more components fail. Levees are predominantly series systems; if one section of a levee fails the system has failed. However, the state of any system depends on the definition of failure. Consider the Bay Delta's function of protecting land against flooding; if any section of levee on any island fails the system has failed. For its function as a water distribution hub, saltwater contamination of fresh water that flows through the Bay Delta would constitute failure. There exist scenarios that would require multiple islands to fail in order for saltwater contamination to occur. The behavior of the Bay Delta as a system in such a scenario isn't purely series or parallel, but a combination of both. Here, parallel sub-systems need to be grouped so that the system is a series of parallel sub-systems, or cut sets (Figure 1). By decomposing the system in this way the probability of failure of the cut sets can be estimated and the remaining system can be treated as purely series.

It is a seemingly difficult task to accurately calculate the probability of failure for a single levee reach. Simply defining the length of a single reach is not straightforward. For a complex levee system, even if the assumed number of components and their respective failure probability estimates are accurate, calculating the probability of failure is non-trivial. We will demonstrate that even if the system is entirely parallel or series, quantifying the probability of failure is no small task.

Series Systems. Let us first consider series levee systems (e.g. an island in the Bay Delta). Let event $E_{i}$, denote the failure of the $i^{\text {th }}$ component. As stated before, failure of a series system is achieved if at least one component fails. The probability of failure of a series system is the union of all component failure probabilities. In set theory notion the probability of failure of a series system of $n$ components is: 


$$
P\left(\text { FaiureSystem }_{\text {series }}\right) \quad P\left(\bigcup_{i=1}^{n} E_{i}\right)
$$

The exact solution to the probability of the union of $n$ events can be obtained using the inclusion exclusion rule, equation 2.

$$
P\left(\bigcup_{i 1}^{n} E_{i}\right) \quad \sum_{i 1}^{n} P\left(E_{i}\right)-\sum_{i<j}^{n} P\left(E_{i} E_{j}\right)+=\sum_{i<j<k}^{n} P\left(E_{i} E_{j} E_{k}\right) \ldots(-1)^{n} P\left(E_{i} E_{j} \ldots E_{n}\right)
$$

The inclusion exclusion rule requires knowledge of all individual event probabilities and knowledge of probabilities of intersections of all possible combinations of events. If failure of each event, $E_{i}$, is statistically independent from all other events the probability of all intersections simplify to the product of their individual event probabilities. If failures are mutually exclusive (i.e. events cannot occur simultaneously) all intersection terms dropout. Unfortunately when dealing with levee systems rarely, if ever, are individual component failures statistically independent or mutually exclusive. Mutual exclusiveness of failures is obviously not a realistic assumption. One component failure doesn't rule out the possibility of another. Lack of statistical independence stems from inherent characteristics of levees: materials that levees are built on and with are spatially correlated, as are loads applied to levees (flooding, seismic, etc).
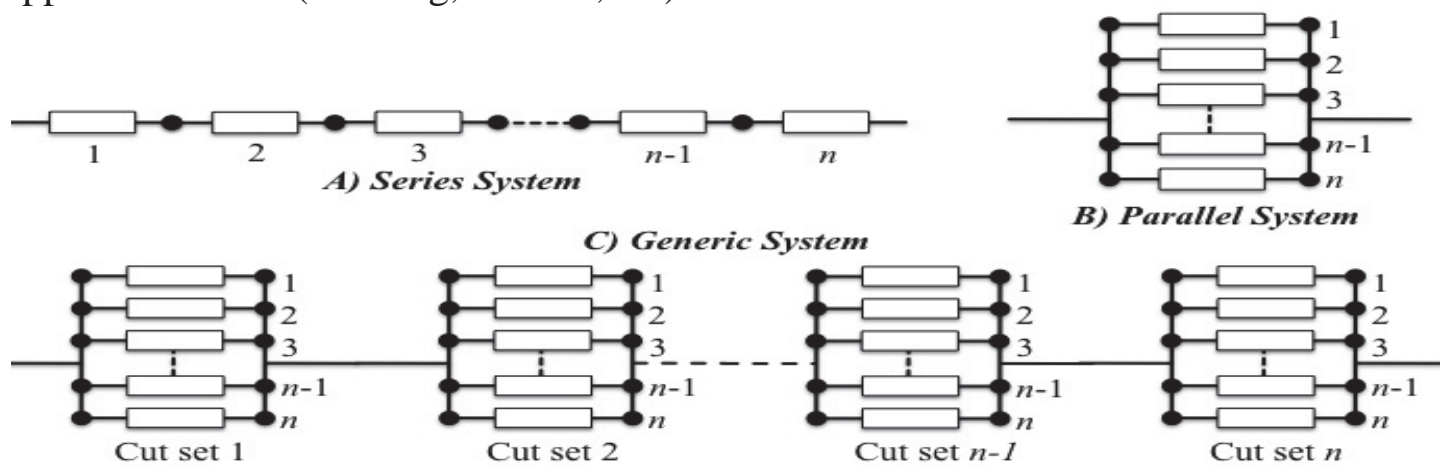

Figure 1: Schematic of different kinds of systems or sub systems.

The exact solution of the union of events lacks practical application. Since no simplifying assumptions apply to levee systems, alternatives are necessary. Bounding the probability of system failure can be a useful tool. Bounds on probability of failure make use of information that is reasonably obtainable, such as uni-, bi-, and tricomponent probabilities of failure. Take an arbitrary system of $n$ components for example. Uni-component probability of failure is that of any individual component, $E_{i}$, bi-component probability of failure is the joint probability of failure of any two components $E_{i} E_{j}$, and similarly tri-component probability of failure is the joint probability of failure of any three components $E_{i} E_{j} E_{k}$. For series systems the narrowest possible uni-component probability bounds are:

$$
\max _{i} P_{i} \leq P\left(\bigcup_{i 1}^{n} E_{i}\right) \leq \min \left(1, \sum_{i=1}^{n} P_{i}\right)
$$

These bounds were derived by Boole (1854) and proven to be the narrowest possible by Fréchet (1935). For large component probability of failure and large number of components (e.g. $P\left(E_{i}\right)>0.05$ and $n>20$ ) the upper bound will reduce to 1 . Having 
an upper bound of 1 for probability failure gives no insight to a specific system. For most practical applications these bounds are too wide. Narrower bounds can be achieved by utilizing higher-order component failure probabilities.

$$
P_{1}+\sum_{i=2}^{n} \max \left(0, P_{i}-\sum_{j 1}^{i-1} P_{i j}\right) \leq P\left(\bigcup_{i 1}^{n} E_{i}\right) \leq P_{1}+\sum_{i=2}^{n}\left(P_{i}-\max _{j<i} P_{i j}\right)
$$

The bounds in equation 4 incorporate both uni-component and bi-component failure probabilities. These bounds were developed through work done by Kounias (1968), Hunter (1976), and Ditlevsen (1979). They have gained wide use (e.g., Song and Der Kiureghian 2003), unfortunately they are dependent on the ordering of bi-component failures. Often, the order that maximizes the lower bound doesn't necessarily minimize the upper bound. Additionally these bounds have not been proven to be the narrowest possible for the information used. With that said, these bounds do offer narrower bounds than equation 3 (Table 1).

Zhang (1993) took the theoretical bounds in 4 and generalized them for still higher-order component failure probabilities. Below, in equation 5, are bounds that utilize tri-component failure probabilities. Bounds that used quad-component failure probabilities were also developed but are not shown here.

$$
\begin{aligned}
& P\left(\bigcup_{i 1}^{n} E_{i}\right) \geq P_{1}+P_{2}-P_{12}+\sum_{i 3}^{n} \max \left(0, P_{i}-\sum_{j 1}^{i-1} P_{i j}+\max _{k \in(1,2, \ldots, i-1)} \sum_{j}^{i-1} P_{1, j \neq k}\right) \\
& P\left(\bigcup_{i 1}^{n} E_{i}\right) \leq P_{1}+P_{2}-P_{12}+\sum_{i 3}^{n}\left[P_{i}-=\max _{k \in\{2,3, \ldots, i-1\}, j<k}\left(P_{i k}+P_{i j}-P_{i j k}\right)\right]
\end{aligned}
$$

Like equation 4 the tri-component bounds also depend on ordering of joint component failures. These bounds are still narrower (Table 1) than the bi-component bounds. It should be apparent that achieving narrower bounds increases computational effort significantly.

Parallel Systems. The exact solution for the failure probability of a parallel system is the intersection of the failure of all its components. Let event $E_{i}$ be the failure of the $i^{\text {th }}$ component. Using set theory notation the probability of failure for a parallel system of $n$ components shown in equation 6. If individual events are statistically independent from one another than this simplifies to equation 7.

$$
\begin{aligned}
& P\left(\text { FailureSystem }_{\text {parallel }}\right) \quad P\left(\bigcap_{i=1}^{n} E_{i}\right) \\
& P\left(\text { Failure System }_{\text {parallel }}\right) \quad P \prod_{i=1}^{n} P\left(E_{i}\right)
\end{aligned}
$$

This results from the fact that the probability of the intersection of statistically independent events is the product of the individual event probabilities (Benjamin and Cornell, 1970). If the events are not statistically independent and the system has a large number of components (e.g., >4) then the probability of their joint occurrence can be difficult and impractical to quantify. As stated earlier, individual component failures in levee systems are rarely statistically independent. In addition, depending on how reach length is defined, the number of components in a levee system can be relatively large. Boole (1854) derived uni-component bounds on the probability of failure of a parallel system: 


$$
\max \left(0, \sum_{i}^{n} P_{i}-(n-1)\right) \leq=P\left(\bigcap_{i}^{n} E_{i}\right) \leq \neq \min P_{i}
$$

These are the narrowest bounds possible if the only available information is unicomponent failure probabilities (Fréchet, 1935). Examining the left side of this inequality for low uni-component probabilities of failure and large n (e.g., $\mathrm{P}\left(\mathrm{E}_{\mathrm{i}}\right)<$ 0.05 and $n>20$ ) the lower bound will often be 0 . A lower bound of 0 for the failure probability of a system is hardly useful. For most practical applications Boole's unicomponent bounds for parallel systems are too wide. There exist no theoretical higher order bounds for parallel systems (Song and Der Kiureghian 2003). However, using De Morgan's Rule (equation 9) higher order bounds can be developed:

$$
P\left(\text { Failure System }_{\text {parallel }}\right) \quad 1-P\left(\bigcap_{i}^{n} E_{i}\right) \quad 1-P\left(\bigcup_{i 1}^{n} \overline{E_{i}}\right)
$$

\section{SPATIAL VARIABILITY AND REACH LENGTH IN LEVEE SYSTEMS}

It is worth noting here that all of the exact solutions and bounds presented above are sensitive to the number of components present in the system. Intuitively, this is expected. Increasing the number of components in a series systems leads to more opportunities for failure. Additional components in parallel systems increases redundancy, which reduces chances of failure. In any case, defining the number of components in a robust repeatable manner is important to the integrity of a probability of failure analysis. Generally in large levee systems, the number of components, or reaches, is inconsistently defined. Either through subjective examination of soil properties and levee geometries (URS, 2008)or with an arbitrary, predetermined, reach length that is not specific to a project or depositional environment (van Manen and Brinkhuis, 2005). Neither of these methods are ideal when considering sensitivity of failure probability estimates to the number of components. Here, we attempt to statistically define the number of components in a levee system based on geotechnical properties that control the probability of failure (e.g., strength, permeability, etc.). Since properties that control failures depend on the failure mode (e.g., seepage failure controlled by permeability, stability failure controlled by strength), reach length should be defined for each failure mode of concern. This concept will make the task of defining levee sections less arbitrary and subjective, and more robust.

Spatial variability is prevalent in geotechnical properties. For example, normalized tip resistance in a layer of sand will vary with depth, or permeability of a sandy layer will vary laterally. A semi-variogram is a tool used to quantify spatial variability. This study utilizes this tool to estimate lateral spatial variability of properties of interest to define levee reach length, and thus number of components in a levee system. Semi-variograms are used prominently in petroleum and mining exploration and have found favor in geotechnical engineering because of their applicability, and ease of use (e.g., Thompson et al. 2007). Semi-variograms are graphical tools that display how much data varies as a function of separation distance. They are used for continuous types of data such as, shear strength, grain size, permeability, etc. Conceptually, they are based on the idea that data collected at two 
relatively close locations is more likely to be similar than data collected at two relatively far away locations.

Semi-variograms plot semi-variance versus separation distance and can generated as experimental semi-variograms (equation 10) or model semi-variograms (equation 11). Experimental semi-variograms are constructed from data pairs, $z_{i}$ and $z_{j}$, sampled at discrete separation distances, $h_{i j}$. Model semi-variograms are continuous functions that describe spatial structure observed in the experimental semi-variogram for all distances, $h$. Experimental semi-variograms need to be constructed from data that exhibits first and second order stationarity. First order stationarity implies that the mean of the sample data doesn't vary with location. Second order stationarity implies that semi-variance is only dependent on separation distance and not absolute location. Functions used for model semi-variograms must be positive definite. There are several reasons for this requirement; the one most relevant to this study is that this maintains positive variance between points (necessary for variance cannot be less than zero).

$$
\begin{aligned}
& \hat{\gamma}(\vec{h}) \frac{1}{2 N(\vec{h})} \sum_{\text {all } i, j, \vec{h}_{i j} \approx \vec{h}}\left[z_{i}-z_{j}\right]^{2} \\
& \gamma(\vec{h}) \quad \frac{1}{2} E\{[Z(\vec{x})-Z(\vec{x}+\vec{h})]\}
\end{aligned}
$$

Figure 2 shows an example of a model semi-variogram. Inspection of the model semi-variogram reveals some general characteristics. At small separation distances the semi-variance is small and increases as separation distance increases. At zero separation distance there is a small offset in the semi-variance. This is known as the nugget effect and is generally attributed to measurement error. There are two categories of model semi-variograms: transitional models, and non-transitional models. In a transitional model, as $h$ increases, semi-variance either, asymptotically approaches a plateau, known as the sill, or reaches a plateau and remains constant. In theory, the sill of a transitional model equals the sample variance of the data set (Clark, 2001). The separation distance at which the sill is reached is referred to as the range. For transitional models that are asymptotic (e.g., the exponential model) the range is defined as the separation distance at which $95 \%$ of the sill is achieved. The model in figure 2 is an example of an asymptotic transitional model. In nontransitional models semi-variance continues to increase with separation distance and does not plateau. Generally speaking, non-transitional models imply that the data used are not stationary on some level.

The range of the model semi-variogram is used to define the reach length of a levee. Range defines the distance at which maximum statistical independence of data is achieved (i.e., distance where correlation is minimized). Data that is separated by distances larger than the range no longer have any spatial correlation. This is not to say that data is not correlated. Rather, correlation is no longer influenced by separation distance. However, for data that is spaced at distances less than the range, correlation is dependent on separation distance. In this case, estimates of how data are related are refined with information provided by the model semi-variogram. 


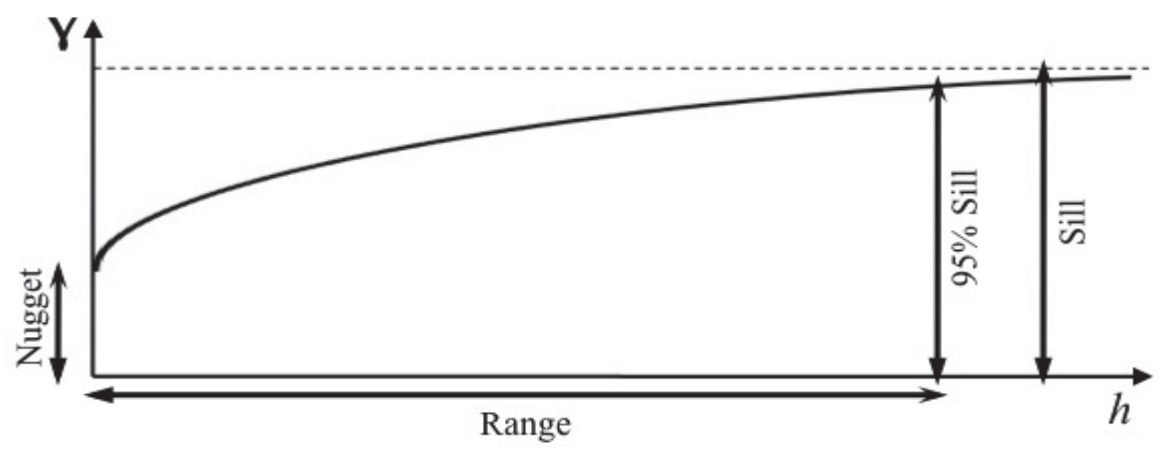

Figure 2: Conceptual diagram of a model semi-variogram. This is a generic, asymptotic transitional model with the sill, range, and nugget labeled.

Example. Consider a single $20 \mathrm{~km}$ long levee. If liquefaction of a sandy layer in the levee foundation is of concern a model semi-variogram could be constructed of tip resistance data in the critical layer. Assume the model reveals a reach length of $800 \mathrm{~m}$ (i.e., 25 components). For the simplicity of the example the following assumptions are made: if liquefaction is triggered in a component it will fail, CSR is a deterministic value and a constant of 0.15 , CRR for all components follows a joint lognormal distribution with identical marginal distributions with mean $=0.25$ and c.o.v. $=0.25$, CRR for components follow a Dennet-Sobel (1995) class correlation matrix. The various bounds on the probability of failure of our example system were calculated using these assumptions and a Dunnet-Sobel (1955) one-dimensional integral to calculate the bi- and tri-component probabilities of failure $\left(P_{i j}\right.$ and $\left.P_{i j k}\right)$. Results are presented in Table 1. The calculations were repeated for two additional cases in which a model semi-variogram reveals reach lengths of $2000 \mathrm{~m}$ and $400 \mathrm{~m}$. This demonstrates sensitivity of bounds to number of components.

Table 1: Sensitivity of number of components and order of probability bounds.

\begin{tabular}{|c|c|c|c|c|c|c|}
\hline Components & \multicolumn{2}{|c|}{$\mathbf{N = 1 0}$} & \multicolumn{2}{c|}{$\mathbf{N}=\mathbf{2 5}$} & \multicolumn{2}{c|}{$\mathbf{N}=50$} \\
\hline Bound & Lower & Upper & Lower & Upper & Lower & Upper \\
\hline Uni & 0.0255 & 0.2550 & 0.0255 & 0.6374 & 0.0255 & 1 \\
\hline Bi & 0.0570 & 0.2112 & 0.0570 & 0.5207 & 0.0570 & 1 \\
\hline Tri & 0.0913 & 0.1861 & 0.0913 & 0.4486 & 0.0913 & 0.886 \\
\hline
\end{tabular}

\section{CLOSING REMARKS}

A conceptual framework of system reliability for levee systems has been discussed. Though bounding the probability of failure is more accessible than an exact solution, in most cases formulas to calculate bounds are still computationally nontrivial and require higher-order failure probabilities (Song and Der Kiureghian, 2003). Higher mode component failure probabilities need to be defined in a manner consistent with the spatial variability. Conceptually, this is straightforward for bicomponent failure probabilities, however, that is not the case for higher-order probabilities. In addition most failure modes depend on more than one variable while semi-variograms are determined from only one variable. Experimental semivariograms can be constructed from data of the property thought to be most critical. Alternatively, experimental semi-variograms can be constructed for all properties effecting failure and the property with the shortest range could control reach length. 


\section{ACKNOWLEDGEMENTS}

This material is based on work supported by the U.S. Department of Homeland Security under Grant Award Number 2008-ST-061-ND0001. Administration of this grant is conducted through the Department of Homeland Security Center of Excellence for Natural Disasters, Coastal Infrastructure and Emergency Management (DIEM). The views and conclusions contained in this document are those of the authors and should not be interpreted as necessarily representing the official policies, either expressed or implied, of the U.S. Department of Homeland Security.

\section{REFERENCES}

Benjamin, J.R., and Cornell, C.A., (1970). Probability, Statistics, and Decision for Civil Engineers. McGraw-Hill

Boole, G. (1854). Laws of thought, American Reprint of 1854 ed., Dover, New York. Clark I. (2001). Practical Geostatistics. Geostokos Limited.

Ditlevsen, O. (1979). "Narrow reliability bounds for structural systems.” Journal of Structural Mechanics, 7(4), 453-472.

Dunnett, C. W., and Sobel, M. (1955). “Approximations to the probability integral and certain percentage points of a multivariate analogue of Student's tdistribution.” Biometrika, 42, 258-260.

Fréchet, M. (1935) “Généralizations du théorème des probabilitéstotales.” Fundamental Mathematics, 25, 379-387.

Hunter, D. (1976). “An upper bound for the probability of a union.” Journal of Applied Probability, 13, 597-603.

Issaks, E. H., and Srivastava, R. M. (1989). An Introduction to Applied Geostatistics. Oxford University Press.

Kounias, E. G. (1968). "Bounds for the probability of a union, with applications." Annuls of Mathematical Statistics, 39(6), 2154-2158.

Moss, R.E.S., Hollenback, J.C., and Ng, J. (2010). "Spatial Variability of Levees as Measured Using the CPT.” Proc. CPT’10, Huntington Beach, CA, May.

Song, J., and DerKiureghian, A. (2003). "Bounds on System Reliability by Linear Programming.” Journal of Engineering Mechanics, 129.6: 627-636.

Thompson, E. M., Baise, L. G., and Kayen, R. E. (2007). "Spatial correlation of shear-wave velocity in the San Francisco Bay Area sediments." Soil Dynamics and Earthquake Engineering, 27, 144-152.

URS. (2008). Delta Risk Management Strategy (DRMS) Phase 1, Risk Analysis Report. Prepared by URS Corporation/Jack R. Benjamin and Associates, Inc., for California Department of Water Resources, December

vanManen, S. E., and Brinkhuis, M. (2005). “Quantitative flood risk assessment for Polders.” Reliability Engineering \& System Safety. 90: 229-237.

Zhang, Y. C. (1993). "High-order reliability bounds for series systems and application to structural systems.” Computers \& Structures, 46(2), 381- 386. 